\title{
Processo de implantação de Programa de Saúde Auditiva em duas maternidades públicas
}

\author{
Implantation process of a Hearing Health Program in two public \\ maternity hospitals
}

\author{
Carmen Barreira-Nielsen ${ }^{1}$, Henrique de Azevedo Futuro Neto ${ }^{2}$, Gilberto Gattaz ${ }^{3}$
}

\begin{abstract}
RESUMO
Objetivos: Apresentar os resultados do processo de implantação de um programa de saúde auditiva neonatal em serviço público e revisar os aspectos determinantes no planejamento, efetividade, manutenção e aprimoramento. Métodos: A fim de colaborar com a implantação de novos serviços, avaliamos os fatores: capacitação de equipe, ambiente de teste (ruído e indivíduo), falso-negativo, evasão e tempo de exame, em um estudo transversal, que incluiu a triagem auditiva de todos os recém-nascidos de duas maternidades públicas, por meio de Emissões Otoacústicas. Resultados: Dos 4951 bebês recém-nascidos no período de 2002 a 2005, foram avaliados $3364(67,9 \%)$, sendo 425 (12,61\%) portadores de indicadores de risco para deficiência auditiva. Houve confirmação da perda auditiva em sete recém-nascidos (0,3\%) por meio do Potencial Evocado Auditivo do Tronco Encefálico. Conclusões: Verificouse a necessidade de protocolos com critério "passa e falha" bem definidos para cada população alvo. A triagem auditiva pode ser realizada até 30 dias após o nascimento, e sua realização não deve se limitar às maternidades. Treinamento e supervisão da equipe são cruciais, além de um sistema de agendamento e acompanhamento dos casos em seguimento, para diminuição da evasão e localização das perdas auditivas de aparecimento tardio, associando os programas de triagem à responsabilidade da confirmação do diagnóstico e intervenção. Desta maneira, teremos condições de mensurar a deficiência auditiva no Brasil e compará-la com o perfil epidemiológico de países onde estes programas já delinearam a saúde auditiva de sua população.
\end{abstract}

Descritores: Triagem neonatal; Recém-nascido; Programas de rastreamento; Emissões otoacústicas espontâneas; Perda auditiva/ diagnóstico; Perda auditiva/prevenção \& controle

\section{INTRODUÇÃO}

A implantação e a discussão de programas de triagem auditiva neonatal têm sido temas constantes de preocupação entre os audiologistas e pediatras. A audição é fundamental para o desenvolvimento da fala e da linguagem. Os estudos dos últimos anos vêm comprovando que a detecção e a intervenção precoces das alterações na audição garantem à criança o desenvolvimento da compreensão e da expressão da lin-

(1) Professora do Centro Universitário Vila Velha - UVV - Vila Velha (ES), Brasil; Pós-graduanda do Programa de Pós-Graduação em Ciências Fisiológicas da Universidade Federal do Espírito Santo - UFES - Vitória (ES), Brasil.

(2) Professor Adjunto da Universidade Federal do Espírito Santo, Centro Biomédico, Departamento de Ciências Fisiológicas - UFES - Vitória (ES), Brasil.

(3) Professor Associado da Pontifícia Universidade Católica de São Paulo PUC-SP - São Paulo (SP), Brasil.

Endereço para correspondência: Carmen Barreira-Nielsen. R. Comissário José Dantas de Melo, 21, Boa Vista, Vila Velha - ES, CEP 29102-770. Email: carmem.barreira@uvv.br

Recebido em: 21/12/2006; Aceito em: 16/3/2007 guagem, comparáveis com as crianças ouvintes da mesma faixa etária.

Em conferência realizada em 1993, o National Institute of Health recomendou a triagem por Emissões Otoacústicas Evocadas (EOA), em todos os recém-nascidos, na ocasião da alta hospitalar, por se tratar de um método bastante eficaz, objetivo, não evasivo e de baixo custo, o que viabiliza a avaliação de um grande número de crianças $^{(1)}$.

Os procedimentos da Triagem Auditiva Neonatal Universal (TANU) recomendados são: o registro das Emissões Otoacústicas Evocadas (EOAEs) ou dos Potenciais Evocados Auditivos do Tronco Encefálico (PEATE). O Comitê Brasileiro sobre Perdas Auditivas na Infância (1999) sugere o uso dos mesmos procedimentos, acrescidos da pesquisa do reflexo cócleo-palpebral (RCP). O registro das Emissões Otoacústicas (EOAS) é o método mais utilizado para a detecção de alterações auditivas de origem coclear. Ao contrário da triagem auditiva comportamental, este método é objetivo, rápido, não invasivo e pode ser realizado em qualquer faixa etária, ressaltando-se sua aplicação em recém-nascidos.

Estima-se que, no Brasil, de três a quatro crianças em 1000 nascem surdas, aumentando para dois a quatro em cada 
100 recém-nascidos $(\mathrm{RN})$ quando provenientes de Unidade de Terapia Intensiva (UTI). Cerca de 50 a 75\% das deficiências auditivas são passíveis de serem suspeitadas no berçário, por meio da triagem auditiva com o registro das Emissões Otoacústicas. De todos os recém-nascidos, 7 a $12 \%$ possuem pelo menos um fator de risco para deficiência auditiva. Em neonatos de risco, cerca de 2,5 a 5\% são portadores de deficiência auditiva, moderada ou severa ${ }^{(2)}$.

Porém, o primeiro diagnóstico tem sido realizado, em média, aos dois anos e meio de idade nos países desenvolvidos; e, no Brasil, a idade média do diagnóstico varia em torno de três a quatro anos ${ }^{(3)}$, podendo levar até dois anos para ser concluído. Isto aponta para a dificuldade em se identificar transtornos auditivos em crianças muito pequenas, resultando, consequientemente, no retardo do diagnóstico e da intervenção terapêutica, na maioria das vezes, em função do falta de integração dos serviços de saúde para o neonato.

A suspeita de deficiência auditiva é feita em $60 \%$ dos casos pelos pais e, em apenas $8 \%$, pelo profissional de saúde. O diagnóstico precoce em crianças é altamente desejável, preferencialmente nos primeiros seis meses de vida, visto que as deficiências auditivas podem levar, a longo prazo, a alterações irreversíveis do processo de aquisição de linguagem e das habilidades cognitivas ${ }^{(4)}$. Portanto, para garantir o acesso da maioria das crianças à intervenção precoce, $\mathrm{o}$ Comitê Brasileiro de Perdas Auditivas na Infância (1999) recomenda a opção de avaliá-las antes da alta da maternidade e, para os nascidos fora do hospital, a avaliação deverá ser feita no máximo até três meses de idade.

Um programa de detecção precoce de surdez deve ser iniciado com a triagem auditiva neonatal, mas deve ser seguido de diagnóstico e reabilitação, contemplando as quatro etapas necessárias para que o programa seja efetivo: rastreamento auditivo e/ou triagem; diagnóstico audiológico; indicação, seleção e adaptação de aparelhos auditivos e re(ha)bilitação auditiva ${ }^{(5)}$. A implantação e a manutenção de um programa de triagem auditiva no Brasil têm acontecido com mais evidência nos últimos cinco anos e contabiliza, até meados de 2006, aproximadamente 250 programas $^{(6)}$, apesar de os primeiros programas de observação comportamental terem aparecido em 1987.

Os métodos de identificação de perda auditiva no período neonatal são atualmente aceitos na prática clínica. Eles são efetivos e espera-se que identifiquem pelo menos $80 \%$ dos casos afetados, podendo apresentar de 2 a $3 \%$ de falsos positivos em programas bem conduzidos ${ }^{(7)}$. Apesar das emissões otoacústicas serem amplamente utilizadas para triagem auditiva neonatal, no Brasil, ainda há muito para se implantar em função dos investimentos em saúde e da extensão territorial. Assim, há de se discutir principalmente a implantação de programa público de saúde auditiva neonatal e revisar os aspectos determinantes no planejamento, efetividade, manutenção e aprimoramento da atenção a saúde auditiva no neonato.

\section{MÉTODOS}

Foram atendidos 4951 recém-nascidos no Programa de Triagem Auditiva Neonatal Universal. Foi realizado um es- tudo transversal, de caráter investigativo, com recém-nascidos de duas maternidades públicas do Estado do Espírito Santo (Vila Velha e Vitória), no período de 2002 a 2005. As duas unidades possuíam Unidades de Tratamento Intensivo e fizeram parte do programa, recém-nascidos provenientes de internações nesta e recém-nascidos do alojamento conjunto com e sem fatores de risco para audição.

A proposta da implantação não se baseou puramente em triagem auditiva, mas teve a preocupação com a promoção de saúde e foi delineada em quatro etapas distintas. A primeira etapa consistiu na aplicação de um questionário investigativo sobre o nível de conhecimento das equipes de saúde, acerca da atuação da Fonoaudiologia, da função auditiva e dos fatores de risco para audição.

O objetivo da segunda etapa foi de tornar a informação compartilhada em todo o hospital e gerar uma rede interligada e fortalecida para detecção precoce da surdez. Por meio de palestra e folheto informativo, foi realizada a capacitação da equipe, principalmente de enfermagem e neonatologia, com relação ao tipo de teste que se propunha na maternidade, público-alvo, condutas e encaminhamentos dentro de uma proposta de saúde integral do neonato.

A terceira etapa envolveu o esclarecimento dos pais ou responsáveis a respeito da audição, do teste e assinatura do termo de consentimento livre e esclarecido para a investigação clínica, aprovados pelo Comitê de Ética e Pesquisa, da Universidade Federal do Espírito Santo com parecer n ${ }^{\circ}$ 022/ 2002.

Foram efetuadas dentro da terceira etapa, 4951 anamneses, com as mães dos recém-nascidos no próprio alojamento conjunto, com o objetivo de colher informações do período gestacional e pós-natal, para localizar RN com Indicadores de Risco para Deficiência Auditiva (IRDA).

Finalmente, a quarta etapa consistiu na realização da triagem auditiva neonatal por meio das Emissões Otoacústicas e pesquisa do reflexo cócleo-palpebral.

Para a realização da triagem auditiva foi utilizado o equipamento Capella (Madsen), conectado a um computador portátil, modelo Itautec compatível com versão Windows, para pesquisa das Emissões Otoacústicas Evocadas (EOAE).

Foi definido, como protocolo, a realização dos seguintes procedimentos:

1) EOA -Transientes: o estímulo utilizado foi o "click não linear", na intensidade de 70 dB NPS, oferecido consecutivamente em ambas orelhas. O modo de aquisição de respostas foi o "Echo-Screen". Foram testadas as frequiências $1 \mathrm{kHz}, 1.5 \mathrm{kHz}, 2 \mathrm{kHz}, 3 \mathrm{kHz}$ e $4 \mathrm{kHz}$.

2) Avaliação comportamental do reflexo cócleo-palpebral (RCP), utilizando o instrumento musical agogô (com estímulo de $90 \mathrm{~dB}$ NPS na faixa de 1 a $3 \mathrm{kHz}$ ).

Os RN foram avaliados no berçário, em época próxima à alta hospitalar e apresentando "falha", foram agendados para retorno em 15 dias para re-teste.

Para a obtenção do "Passa", as emissões otoacústicas transientes foram consideradas presentes se todos os critérios fossem encontrados:

1 Amplitude de resposta acima do ruído em pelo menos uma oitava da banda de freqüência em 3 dB NPS, ampli- 
tude de resposta acima do ruído igual ou superior a $6 \mathrm{~dB}$ NPS, nas demais bandas de frequiências; reprodutibilidade para bandas de freqüências e geral igual ou superior a $70 \%$ em pelo menos três freqüências a partir de $2 \mathrm{kHz}$;

2 Estabilidade da sonda inspecionada visualmente pelo marcador "fit".

Todos os RN que também falharam no re-teste, foram encaminhados para avaliação diagnóstica por intermédio do Potencial Evocado Auditivo do Tronco Encefálico (PEATE), imitanciometria e consulta otorrinolaringológica, na Policlínica de Fonoaudiologia do Centro Universitário Vilha Velha (UVV). Este trabalho não tem como objetivo descrever os exames realizados fora da maternidade; desta forma, apenas quantificará a alteração auditiva, confirmada no centro diagnóstico proveniente do programa de saúde auditiva implantado.

Aqueles pacientes com perdas auditivas confirmadas foram encaminhados para acompanhamento pela equipe da Policlínica de Referência da UVV para seleção e adaptação de prótese e re(ha)bilitação auditiva na própria instituição.

Aqueles RN que passaram na triagem, mas apresentaram IRDA, também foram orientados para um acompanhamento semestral e com duração de três anos, utilizando-se re-teste das Emissões Otoacústicas e avaliação do desenvolvimento cognitivo, motor e psíquico.

Todos os dados coletados foram transpostos para análise estatística, a fim de quantificar as variáveis da implantação do programa, nas maternidades pesquisadas, apresentando apenas os percentuais e valores absolutos. A pesquisa teve enfoque indutivo e estatística descritiva de organização de dados foi utilizada, sendo desnecessária uma análise inferencial, em função dos próprios objetivos do estudo.

\section{RESULTADOS}

No período de agosto 2002 a agosto de 2005, foram registrados 4951 nascimentos nas duas maternidades. A amostra foi composta por RN sem indicadores de risco para deficiência auditiva e por outros com indicadores; destes, locali- zamos $425(12,6 \%)$ RN que apresentavam pelo menos um fator de risco. Na Tabela 1, está exposta a proporção como cada indicador apareceu na amostra.

A implantação do programa foi realizada em duas maternidades públicas, A (2002/2003 e 2003/2004) e B (2004/ 2005), que têm número de nascimentos bem distintos. $\mathrm{Na}$ Tabela 2, visualiza-se a diferença entre as unidades ao longo dos anos e, sobretudo, é destacado o índice de aceitação e efetividade ao programa com o passar destes. Os recém-nascidos com alta no final de semana e/ou feriados não foram avaliados e receberam orientação para realização externa do procedimento. Na Tabela 2, este grupo, pode ser localizado pelo título "outros não avaliados".

A relação "passa/falha" de todos os RN avaliados, re-teste e evasão é mostrada na Tabela 3, que apresenta os valores em diferentes períodos do programa e suas especificidades.

$\mathrm{Na}$ Tabela 4, constata-se que a alteração auditiva foi confirmada na amostra por meio do PEATE, nos grupos com e sem indicadores de risco para deficiência auditiva na mesma proporção. Verifica-se, também, percentual de falso-positivo para as variáveis grupo e gênero.

Todos os pacientes que tiveram como resultado "falha" no re-teste ou "passa", mas apresentavam IRDA, foram en-

Tabela 1. Fatores de risco mais prevalentes encontrados em 425 recémnascidos com Indicadores de Risco para Deficiência Auditiva (IRDA) do Programa de Triagem Auditiva Neonatal

\begin{tabular}{lcc}
\hline Fatores de risco & $\mathrm{n}$ & $\%$ \\
\hline Medicação ototóxica & 92 & $21,6 \%$ \\
Peso ao nascimento < 1500 g & 89 & $21,0 \%$ \\
Ventilação mecânica por período > 5 dias & 85 & $20,0 \%$ \\
Infecção congênita & 59 & $13,8 \%$ \\
Hiperbilirrubinemia em nível de exsangüíneo transfusão & 45 & $10,5 \%$ \\
História familiar de deficiência auditiva congênita & 40 & $9,5 \%$ \\
Apgar de 0-4 no 1 minuto & 30 & $7,0 \%$ \\
Síndromes associadas à deficiência auditiva & 14 & $3,3 \%$ \\
Meningite bacteriana & 11 & $2,6 \%$ \\
Anomalias crânio-faciais & 4 & $0,9 \%$ \\
\hline
\end{tabular}

Tabela 2. Apresentação do número de nascidos vivos/ano registrado nas unidades em que foram implantados os programas de TAN x porcentagem de avaliados

\begin{tabular}{lcccc}
\hline Ano & Registro & Avaliados & Não aceitação & Outros não avaliados \\
\hline $2002 / 2003$ & 970 & $911(94 \%)$ & $12(1,2 \%)$ & $47(4,8 \%)$ \\
$2003 / 2004$ & 869 & $834(96 \%)$ & $5(0,6 \%)$ & $30(3,5 \%)$ \\
$2004 / 2005$ & 3112 & $1619(52 \%)$ & 0 & $1493(48 \%)$ \\
\hline Total & 4951 & $3364(68 \%)$ & $17(0,34 \%)$ & $1570(31,7 \%)$ \\
\hline
\end{tabular}

Tabela 3. Tabela de referência "Passa" e "Falha"

\begin{tabular}{lccccccc}
\hline Ano & Triados & $1^{\circ}$ teste Passa & $1^{\circ}$ Falha & Reteste & Evasão & $2^{\circ}$ teste Passa & $2^{\circ}$ Falha \\
\hline $2002 / 2003$ & 911 & $792(87 \%)$ & $119(13 \%)$ & $45(37,8 \%)$ & $74(62 \%)$ & $39(86,6 \%)$ & $6(0,66 \%)$ \\
$2003 / 2004$ & 834 & $742(89 \%)$ & $92(11 \%)$ & $53(58 \%)$ & $39(42 \%)$ & $45(85 \%)$ & $4(0,48 \%)$ \\
$2004 / 2005$ & 1619 & $1487(91,9 \%)$ & $132(8,1 \%)$ & $86(65 \%)$ & $46(35 \%)$ & $77(89,5 \%)$ & $9(0,56 \%)$ \\
\hline Total & 3364 & $3021(89,3 \%)$ & $343(10 \%)$ & $184(53,6 \%)$ & $159(46,3 \%)$ & $161(87 \%)$ & $19(0,57 \%)$ \\
\hline
\end{tabular}


Tabela 4. Prevalência de alteração auditiva segundo os testes PEATE e EOA x gênero

\begin{tabular}{lcccc}
\hline Recém-nascidos sem IRDA“Falha" EOA & PEATE alterado DANS & Sexo feminino & Sexo masculino \\
\hline $\mathrm{n} 2939(87,4 \%)$ & $8(0,27 \%)$ & $5(62 \%)$ & $1(20 \%)$ & $4(80 \%)$ \\
$\mathrm{n} 425(12,6 \%)$ & $11(2,6 \%)$ & $7(63 \%)$ & $4(57 \%)$ & $3(43 \%)$ \\
\hline
\end{tabular}

Legenda: PEATE - Potencial Evocado do Tronco Encefálico; EOA - Emissões Otoacústicas Evocadas; DANS - Deficiência Auditiva Neurossensorial

Tabela 5. Número de Recém-nascidos (RN) "passa" com Indicadores de Risco para Deficiência Auditiva (IRDA) que procuraram a Policlínica de Referência para dar continuidade aos atendimentos

\begin{tabular}{lccc}
\hline Ano & RN IRDA & Seguimento & Evasão \\
\hline $2002 / 2003$ & $145(15,9 \%)$ & $64(44 \%)$ & $81(56 \%)$ \\
$2003 / 2004$ & $111(13,3 \%)$ & $76(68 \%)$ & $35(32 \%)$ \\
$2004 / 2005$ & $169(10,4 \%)$ & $30(18 \%)$ & $139(82 \%)$ \\
\hline Total & $425(12,6 \%)$ & $170(52,6 \%)$ & $311(73,3 \%)$
\end{tabular}

Tabela 6. Duração média da triagem auditiva bilateral através das Emissões Otoacústica Evocadas Transientes em recém-nascidos na alta x reteste

\begin{tabular}{lll}
\hline & $\begin{array}{l}\text { Duração (seg)/alta } \\
\text { Orelha direita/esquerda ( } \mathrm{n}=184 \mathrm{RNs})\end{array}$ & $\begin{array}{l}\text { Duração (seg)/retorno } \\
\text { Orelha direita/esquerda ( } \mathrm{n}=184 \mathrm{RNs})\end{array}$ \\
\hline 2002 a 2005 & $105 \mathrm{~s}$ & $92 \mathrm{~s}$ \\
\hline
\end{tabular}

caminhados para diagnóstico e acompanhamento. Na Tabela 5, são identificados os índices de RN em acompanhamento e de evasão no seguimento.

A fim de verificar a viabilidade da execução do teste logo após o nascimento, estes resultados foram comparados com os realizados após uma semana de vida, considerando o tempo médio em segundos para obtenção de resposta por Emissões Otoacústicas Evocadas Transientes no teste e no re-teste (Tabela 6).

\section{DISCUSSÃO}

Hipotetizando que a integração com o corpo de profissionais que já estavam inseridos seria determinante para a implantação do programa, aplicou-se um questionário à equipe, que apontou para a falta de conhecimento em três questões principais: atuação da Fonoaudiologia na maternidade, fatores de risco para a audição e a importância da detecção precoce da deficiência auditiva. Com este achado, foi estabelecida uma capacitação e um relacionamento mais próximo à equipe de saúde e administrativa da unidade.

O programa iniciou com um índice de aceitação dos pais (Tabela 2) inferior à situação atual. Identificamos que o tempo de programa trouxe diminuição sistemática no índice de não aceitação dos pais em realizar a triagem e, certamente, podemos justificar este dado ao comprometimento e conhecimento da própria equipe capacitada sobre a importância do teste, como também o efeito da divulgação. O tempo de permanência da parturiente e do RN nas maternidades impediu que houvesse tempo disponível para algum esclarecimento sobre o teste antes do parto.

Verificamos, por meio da anamnese, que apenas $32 \%$ das gestantes se submeteram as consultas de pré-natal por mais de seis vezes e, ainda, $7 \%$ das gestantes não realizaram o pré-natal. O menor número de consultas no pré-natal pode estar associado a um início mais tardio, ou a dificuldades de acesso aos serviços de saúde. Entre 32\% de entrevistadas com mais de seis consultas, não verificamos conhecimento sobre os fatores de risco para a audição e triagem auditiva, o que justifica também a capacitação das equipes de atenção básica de saúde.

Para avaliar a efetividade do programa, foi realizada a verificação dos indicadores de qualidade, os quais se referem à porcentagem de recém-nascidos avaliados versus número de nascidos vivos da maternidade, nos anos em que os programas foram aplicados nas duas maternidades. Os valores oscilaram entre 94\%, 96\%, 52\% de RN avaliados. Um programa, para ser efetivo, deve contemplar pelo menos $90 \%$ dos nascimentos da unidade ${ }^{(6)}$. Foram encontrados entraves administrativos para a execução diária da triagem auditiva em uma das maternidades.

Dos 4951 recém-nascidos no período de 2002 a 2005 , foram avaliados 3364 (68\%), sendo que 425 (12,6\%) apresentavam IRDA, valor semelhante à literatura encontrada ${ }^{(3)}$, e $11(2,5 \%)$ apresentaram mais que um IRDA. Por meio dos questionários, pode-se verificar que, quando os pediatras já alertam sobre a importância do teste, os pais o valorizam mais e o procuram no período da alta.

Como fatores de risco auditivos mais prevalentes, constatamos: peso ao nascimento abaixo de $1500 \mathrm{~g}$ (21\%); medicação ototóxica $(21 \%)$ e ventilação mecânica por período superior a cinco dias (20\%), dados estes, concordantes com os encontrados na literatura ${ }^{(8-9)}$. Do total de 3364 de RN avaliados, $343(10,7 \%)$ foram reprovados no primeiro teste antes da alta hospitalar. Determinar o critério de "passa" em programas de triagem é um fator importante, pois afeta a operacionalização de todas as etapas. No presente trabalho, buscou-se determinar que a amplitude de resposta estivesse 
pelo menos de $3 \mathrm{~dB}$ a $6 \mathrm{~dB}$ acima do ruído nas bandas de freqüências de 1 a $4 \mathrm{kHz}$ e, também, garantir que todos que falhassem pudessem retornar para o re-teste.

Do total de "falha", apenas $184(53 \%)$ retornaram para o re-teste, número inferior aos $70 \%$, apontados como índice mínimo de retorno para seguimento de triagem ${ }^{(10)}$. Diante de $47 \%$ de evasão, sugere-se controle efetivo de variáveis como ambiente, equipamento e prática na execução, para se diminuir o re-teste. Acredita-se, também, em uma possível associação do grau de instrução ao grau de evasão destas parturientes provenientes de maternidades públicas.

Dos recém-nascidos que apresentaram IRDA e passaram na triagem, 425 foram encaminhados para seguimento do desenvolvimento até três anos, porém apenas 170 (52,6\%) RN compareceram ao programa pelo menos para o primeiro atendimento e $44(10,3 \%)$ até o segundo ano (Tabela 5), índice bem inferior ao encontrado na literatura ${ }^{(11)}$; no entanto, com uma característica de aumento com o passar dos anos Entretanto, no período entre 2004/2005, houve um aumento significativo na evasão; supõe-se que isso ocorreu em função da distância maior entre o Centro de Diagnóstico e a Maternidade B. A perda destes pacientes pode ser atribuída, também, à dificuldade ou inadequação dos sistemas de agendamento, falta de contato telefônico, dificuldade de transporte; mas, principalmente, pela falta de comunicação entre a família e a equipe.

Destaca-se a importância do seguimento não estar atrelado apenas a maternidades ou centro de especialidades, mas também a rotinas de saúde infantil, uma vez que a falta de acompanhamento impede a detecção de perdas auditivas tardias e subestima os falso-negativos. Há necessidade de métodos de triagem adicionais talvez nas escolas, já que, aproximadamente, $25 \%$ de perdas auditivas bilaterais aparecem no pós-natal e, muitas vezes, sem indicadores de risco auditivo ou aparecendo depois de três anos ${ }^{(12)}$.

Dos RN que retornaram para re-teste, 19 (1,7\%) mantiveram a "falha" (Tabela 3). Para a confirmação da perda auditiva, foram encaminhados ao diagnóstico, por meio do Potencial Evocado Auditivo do Tronco Encefálico e não houve evasão para conclusão do diagnóstico, confirmando alteração auditiva em $12 \mathrm{RN}$.

A idade média do fechamento do diagnóstico foi de quatro meses e meio, tempo superior ao preconizado ${ }^{(2.5-13)}$ e a idade de intervenção, ainda sem aparelho auditivo, foi de seis meses, adquirindo em média o sistema de amplificação com 11 meses. O estudo reflete o panorama nacional no qual a maioria dos programas de triagem se encontra, ou seja, não interligados a um programa de diagnóstico e reabilitação, como sugerido ${ }^{(14)}$. Entretanto, nossos dados diferem dos dados encontrados na literatura nacional, que apresentam tempo perdido de mais de dois anos entre a suspeita e a confirmação da $\mathrm{DA}^{(15)}$.

Foi confirmada a deficiência auditiva neurossensorial bilateral, em $7(0,2 \%)$ RN, $3(0,08 \%)$ RN com alterações de orelha média e $2(0,05 \%)$ RN confirmaram normalidade depois do sexto mês de vida. O RCP esteve ausente em todos os recém-nascidos com confirmação de deficiência auditiva e a incidência de perda auditiva nesta população esteve em $0,3 \%$.
Ao compararmos a incidência de deficiência auditiva em RN com IRDA e sem IRDA, verificamos não ser estatisticamente significante, aparecendo na mesma proporção. Isso permite ao programa estabelecer o mesmo protocolo de avaliação para os dois grupos. Entre os recém-nascidos que confirmaram deficiência auditiva, $4(0,1 \%)$ representaram o grupo de RN pré-termo e com baixo peso, o que aponta para coerência com a literatura que mostra que idade gestacional inferior a 31 semanas e baixo peso devem ser investigados ${ }^{(16)}$ e requerem intervenções especiais.

Os achados apontaram para um resultado de 7 (6,3\%) RN falso-positivos, sendo que $4(58,9 \%)$ do grupo de risco e $3(41 \%)$ do grupo sem risco. O resultado falso-positivo no teste de emissões otoacústicas decorre freqüentemente da presença de restos mesenquimais na orelha média ${ }^{(17)}$. O elevado número de falha no primeiro teste pode ser atribuído ao número elevado de prematuros ( $\mathrm{n}=392$ neste estudo) e baixo peso $(n=79)$ e, em seguida, devido a afecções de orelha média, retardo na maturação coclear e na mielinização das vias auditivas $^{(18)}$. A incidência de registro das emissões otoacústicas relacionadas ao peso dos recém-nascidos é de $72 \%$, nos bebês com peso entre $1500 \mathrm{~g}$ e $2500 \mathrm{~g}$ e de $55 \%$, nos bebês com peso inferior a $1500 \mathrm{~g}^{(19)}$.

Em nossa experiência, acredita-se em menor índice de falso-positivos a partir do quarto dia de vida ${ }^{(20-21)}$. Já foi encontrado, em estudo anterior ${ }^{(22)}$ que, no segundo dia de vida, a estabilidade foi significantemente melhor e com uma melhor simetria $(\mathrm{p}<0.001)$ e menos incidência de artefatos $(\mathrm{p}<0.001)$. Além disso, a maioria dos estudos é unânime sobre a existência de maiores amplitudes nas respostas das primeiras 24 horas. Outro fator que está diretamente ligado ao alto índice de falso-positivos é a escolha equivocada do protocolo e da tecnologia para a população-alvo. Um resultado falso-positivo tem um efeito muito maior do que se pode mensurar com o tempo extra, reprogramando e re-testando desnecessariamente um RN, o que normalmente "incha" o programa; todavia, há de se refletir sobre o efeito de um falso-positivo na relação familiar.

Entre os recém-nascidos com confirmação de alteração auditiva bilateral, 7 (58\%) foram RN do sexo masculino. Não localizamos estudos que comprovem a predominância de deficiência auditiva relacionada ao gênero; entretanto, a resposta auditiva é maior no gênero feminino e na orelha direi$\mathrm{ta}^{(23)}$. Talvez isto se deva ao número de células ciliadas maior no gênero feminino(24), gênero que também apresenta maior prevalência de emissões otoacústicas ${ }^{(25)}$.

O trabalho desenvolvido em maternidade pública tem suas especificidades, que muitas vezes, alteram o fluxo dos atendimentos, entre elas o quadro de funcionários restrito, grande número de partos/dia, tempo disponível para capacitação dentro e fora da unidade e articulação da maternidade com outros serviços do próprio SUS, que disponham de diagnóstico e intervenção, principalmente no que se refere à concessão do aparelho de amplificação sonora, conforme preconizado ${ }^{(5)}$.

O espaço físico foi sinalizado, também, como um complicador pela ausência de salas disponíveis, e o tipo predominante de alojamento conjunto com até seis parturientes dificultou a execução do procedimento em condições favo- 
ráveis. As triagens foram realizadas predominantemente fora do alojamento conjunto, pois o ruído dos próprios recémnascidos e, sobretudo, a circulação de outros profissionais inviabilizaram o procedimento no alojamento. $\mathrm{O}$ ambiente comum utilizado para triagem auditiva pode interferir no registro das respostas das emissões otoacústicas ${ }^{(26)}$. Desse modo, devem ser reconsideradas as alternativas de aquisição de resposta e mudança de protocolo. Foi importante também considerar a hora da mamada, do banho e das visitas para minimizar as variáveis.

Constatou-se que, mesmo minimizando os fatores ambientais e do equipamento, foi significativo o ruído de fundo, provavelmente do estado da orelha média e/ou externa, e quando da respiração, sucção e deglutição. Em RN cooperantes, o ruído é concentrado na faixa de frequiência baixa ${ }^{(27)}$; crianças na faixa de zero a um mês têm relação sinal-ruído menor quando comparadas a crianças mais velhas ${ }^{(28)}$.

Outro fato que não só está relacionado ao ruído, mas também ao tempo de nascido, foi analisado em dois momentos (Tabela 6): o tempo de exame. Foi possível identificar que, em média, o tempo de exame se aproximou da literatura $\left(82 \mathrm{~s}^{(29)} 89 \mathrm{~s}^{(30)}\right)$; porém, foi maior na triagem inicial quando comparado ao tempo de re-teste na própria maternidade com idades entre 15 e 30 dias de vida. Os achados diferem dos estudos realizados com retornos em ambulatórios com idades superiores ${ }^{(20)}$. Neste estudo, pretende-se justificar que o RN nascido, ainda na alta, possivelmente tende a apresentar resíduos do nascimento na orelha externa e/ou média, o que não impede que a resposta seja "passa", mas proporciona um tempo maior para localizar a resposta.

\section{CONCLUSÃO}

Constatou-se que o tempo de execução da proposta per- mitiu delinear alguns aspectos determinantes no planejamento, efetividade, manutenção e aprimoramento do programa de saúde auditiva no neonato. Primeiro, há necessidade de protocolos com critério "passa e falha" e tecnologia bem definida para a população-alvo, já que isto diminui o falsopositivo.

Considerar que a triagem auditiva possa ser realizada até 30 dias após o nascimento, não limitando sua realização às maternidades, mas que seja oferecido também nos serviços de nível primário como em programas de puericultura, sem perder a eficiência, posto que o status "falha" é mais baixo após o quarto dia.

Conscientização, treinamento e supervisão da equipe e, principalmente, um sistema de agendamento e monitoramento eletrônico dos seguimentos para diminuição da evasão, agregando um sistema de apoio a famílias, por meio da enfermagem, neonatologia e ginecologia, que fortaleça o vinculo com o seguimento. Sugere-se integração com o PSF, já existente em muitas unidades de saúde, para a localização das perdas auditivas de aparecimento tardio e conclusão do diagnóstico no tempo pré-estabelecido.

As alterações auditivas foram localizadas e confirmadas; entretanto, mesmo com a evolução dos equipamentos e métodos, tem-se ainda um desafio: localizar as deficiências auditivas leves e, ainda, aquelas pouco freqüentes como, por exemplo, de frequiência isolada e localização de falso-negativos.

Tem-se tornado cada vez mais evidente que a iniciativa de implantar programas de TANU deve agregar a responsabilidade de confirmação diagnóstica/intervenção e que seja integrada a programas de atenção a saúde do neonato, pois assim haverá condições de mensurar a deficiência auditiva no Brasil e compará-la com o perfil epidemiológico de países onde estes programas já delinearam sua saúde auditiva.

\begin{abstract}
Purpose: To present the results of an implantation process of a neonatal hearing health program in public services and to review determinant aspects on its planning, effectiveness, maintenance and improvement. Methods: In order to aid the implementation of new services, factors such as team qualification, test environment (noise and subject), false-negative, evasion and evaluation time were assessed in a cross-sectional study which included the hearing screening of all newborns in two public maternity hospitals, through otoacoustic emissions. Results: Out of 4951 newborns in the period from 2002 to 2005, 3364 (67.9\%) were assessed; 425 $(12.61 \%)$ had risk indicators for hearing impairment. Hearing loss was confirmed in seven newborns $(0.3 \%)$ through the Brainstem Auditory Evoked Potential. Conclusions: The need for protocols with well defined "pass and fail" criterion for each target population was verified. Hearing screening should be carried out within 30 days following birth and should not be limited to maternity hospitals. Team training and supervision is crucial, as well as electronic appointment and monitoring system, in order to decrease evasion and to detect late hearing loss, associating screening programs with the responsibility of diagnostic confirmation and intervention. This way, one will be able to measure hearing deficiency in Brazil and compare it with epidemiological profiles of countries where such programs have already outlined the hearing health of their population.
\end{abstract}

Keywords: Neonatal screening; Infant, newborn; Mass screening; Otoacoustic emissions, spontaneous; Hearing loss/diagnóstico; Hearing loss/prevention \& control 


\section{REFERÊNCIAS}

1. National Institutes of Health Consensus Development Conference Statement. Early identification of hearing impairment in infants and young children. Int J Pediatr Otorhinolaryngol. 1993;27(3):215-27. Review.

2. INES - Instituto Nacional de Educação de Surdos. Prevenção. [citado 2006 Out 10]. Disponível em: http://www.ines.org.br/Paginas/ prevencao.asp 14.10.2006.

3. Audiologic screening of newborn infants who are at risk for hearing Impairment. ASHA. 1989;31(3):89-92.

4. Lamprecht, A. Evozierte otoakustische emissionen bel normallhoerenden und schwerhorigen erwachsenen und kindern. Laryngo-Rhino-Otho. 1991;70:1-4.

5. Joint Committee on Infant Hearing. 1994 position statement. Audiol Today. 1995;6: 6-9.

6. GATANU - Grupo de Apoio à Triagem Auditiva Neonatal Universal [texto na Internet]. [citado 2006 Out 10]. Disponível em: http:// www.gatanu.org/.

7. The European consensus statement on neonatal hearing screening Finalized at the European Consensus Development Conference on Neonatal Hearing Screening May 1998. Milan, Italy. Scand J Audiol. 1998;27(4):259-60. Review.

8. Manfredi AKS, Santos CB. Intervenção fonoaudiológica junto às mães ou responsáveis por recém-nascidos de risco para deficiência auditiva, precocemente detectada. Medicina (Ribeirão Preto). 2002;35(1):70-7.

9. Barreira-Nielsen CSC, Posses P. Triagem auditiva neonatal: estudo da incidência de indicadores de risco [monografia]. Vila Velha: Centro Universitário Vila Velha 2005.

10. Prieve BA, Falter SR. COAEs and SSOAEs in adults with increased age. Ear Hear. 1995;16(5):521-8

11. Weichbold V, Welzi-Mueller K. Maternal concern about positive test results in universal newborn hearing screening. Pediatrics. 2001;108(5):1111-6.

12. Weichbold V, Nekahm-Heis D, Welzi-Mueller K. Universal newborn hearing screening and postnatal hearing loss. Pediatrics. 2006;117(4):e631-6

13. Joint Committee on Infant Hearing; American Academy of Audiology; American Academy of Pediatrics; American Speech-Language-Hearing Association; Directors of Speech and Hearing Programs in State Health and Welfare Agencies. Year 2000 position statement: principles and guidelines for early hearing detection and intervention programs. Joint Committee on Infant Hearing, American Academy of Audiology, American Academy of Pediatrics, American Speech-Language-Hearing Association, and Directors of Speech and Hearing Programs in State Health and Welfare Agencies. Pediatrics. 2000;106(4):798-817.

14. Comitê Brasileiro sobre Perdas Auditivas na Infância. Recomendação 01/99 do Comitê Brasileiro sobre perdas auditivas na infância. J Cons Fed Fonoaudiol. 2000;5:3-7.

15. Nóbrega M. Estudo da deficiência auditiva em crianças e adolescentes, comparando-se os períodos de 1990 a 1994 e 1994 a 2000 [tese]. São Paulo: Universidade Federal de São Paulo. Escola Paulista de Medicina; 2004.
16. Watkin PM, Baldwin M, McEnery G. Neonatal at risk screening and the identification of deafness. Arch Dis Child. 1991;66(10 Spec No): $1130-5$

17. Doyle KJ, Burggraaff B, Fujikawa S, Kim J, MacArthur CJ. Neonatal hearing screening with otoscopy, auditory brain stem response, and otoacoustic emissions. Otolaryngol Head Neck Surg. 1997;116(6 Pt 1):597-603

18. Hall JW. Handbook of otoacoustic emissions. San Diego, Calif.: Singular; c2000.

19. Gattaz G, Santos FA, Mahl GS. Incidência do registro de EOA relacionadas ao peso dos recém-nascidos. Distúrb Comun. 2001;12(2):173-84.

20. Korres S, Nikolopoulos T, Ferekidis E, Gotzamanoglou Z, Georgiou A, Balatsouras DG. Otoacoustic emissions in universal hearing screening: which day after birth should we examine the newborns? ORL J Otorhinolaryngol Relat Spec. 2003;65(4):199-201.

21. Trinidad Ruiz G, Pantoja Hernandez CG, Trinidad Ramos G, Serrano Berrocal MA, Pardo Romero G, Gonzalez Palomino A, Blasco Huelva A. [Controlling retests in a universal hearing screening program]. Acta Otorrinolaringol Esp. 2005;56(3):96-101. Spanish.

22. Del Buono ZG, Mininni F, Delvecchio M, Pannacciulli C, Mininni S. [Neonatal hearing screening during the first and second day of life]. Minerva Pediatr. 2005;57(4):167-72. Italian.

23. Kannnan PM, Lipscomb DM. Letter: Bilateral hearing asymmetry in a large population. J Acoust Soc Am. 1974;55( 5):1092-4.

24. Wright A, Davis A, Bredberg G, Ulehlova L, Spencer H, Bock G, et al. Hair cell distributions in the normal human cochlea. A report of a European working group. Acta Otolaryngol Suppl. 1987;436:15-24.

25. Cassidy JW, Ditty KM. Gender differences among newborns on a transient otoacoustic emissions test for hearing. J Music Ther. 2001;38(1):28-35.

26. Headley GM, Campbell DE, Gravel JS. Effect of neonatal test environment on recording transient-evoked otoacoustic emissions. Pediatrics. 2000;105(6):1279-85.

27. Glattke TJ, Robinette MS. Transient evoked otoacoustic emissions. In: Robinette MS, Glattke TJ, editors. Otoacoustic emissions: clinical applications. 2nd ed. New York: Thieme; 2002. p. 95-115.

28. Kappor R, Panda NK. Transient evoked otoacoustic emissions. Indian J Pediatr. 2006;73(4):283-6.

29. Aidan D, Lestang P, Avan P, Bonfils P. Characteristics of transientevoked otoacoustic emissions (TEOES) in neonates. Acta Otolaryngol. 1997;117(1):25-30

30. Raineri GG, Coube CZV, Costa Filho O A, Alvarenga KF. Emissões otoacústicas evocadas - produto de distorção em neonatos audiologicamente normais. Rev Bras Otorrinolaringol. 2001;67(5). 